\title{
Predicting the Consumption Speed of a Premixed Flame Subjected to Unsteady Stretch Rates
}

\author{
Meysam Sahafzadeh \\ Department of Mechanical and Industrial Engineering, Ryerson University, 350 Victoria St., \\ Toronto, Ontario, Canada M5B 2K3 \\ Seth B. Dworkin \\ Department of Mechanical and Industrial Engineering, Ryerson University, 350 Victoria St., \\ Toronto, Ontario, Canada M5B 2K3 \\ Larry W. Kostiuk \\ Department of Mechanical Engineering, University of Alberta, 116 St. and 85 Ave., \\ Edmonton, AB, Canada T6G 2R3
}

Corresponding Author: Seth Benjamin Dworkin

Department of Mechanical and Industrial Engineering, Ryerson University, 350 Victoria St., Toronto, Ontario, Canada M5B 2K3

Email: seth.dworkin@ryerson.ca

Phone: 1 (416) 979-5000 x7311

Fax: 1 (416) 979-5265

\section{Full-length article}




\section{Abstract}

The stretched laminar flame model provides a convenient approach to embed realistic chemical kinetics when simulating turbulent premixed flames. When positive-only periodic strain rates are applied to a laminar flame there is a notable phase lag and diminished amplitude in heat release rate. Similar results have being observed with respect to the other component of stretch rate, namely the unsteady motion of a curved flame when the stretch rates are periodic about zero. Both cases showed that the heat release rate or consumption speed of these laminar-premixed flames vary significantly from the quasi-steady flamelet model. Deviation from quasi-steady behaviour increases as the unsteady flow time scale approaches the chemical time scale that is set by the stoichiometry. A challenge remains in how to use such results predictively for local and instantaneous consumption speed for small segments of turbulent flames where their unsteady stretch history is not periodic.

This paper uses a frequency response analysis as a characterization tool to simplify the complex nonlinear behaviour of premixed methane air flames for equivalence ratios from 1.0 down to 0.7 , and frequencies from quasi-steady up to $2000 \mathrm{~Hz}$ using flame transfer functions. Various linear and nonlinear models were used to identify appropriate flame transfer functions for low and higher frequency regimes, as well as extend the predictive capabilities of these models. Linear models were only able to accurately predict the flame behaviour below a threshold of when the fluid and chemistry time scales are the same order of magnitude. Other proposed transfer functions were tested against arbitrary multi-frequency stretch inputs and were shown to be effective over the full range of frequencies.

Keywords: Laminar Premixed Flames, Transient Response, Stretch Rate, Consumption Speed, Burning rate, Linear and Nonlinear Systems, Frequency Response Analysis 


\section{Introduction}

Understanding turbulent combustion is important due to its common occurrence in industrial applications such as internal combustion engines and furnaces. The challenges related to predicting turbulent combustion behavior have been discussed extensively in terms of their time-dependent and multidimensional nature, as well as having a large range of hydrodynamic time and length scales interacting with these scales of the chemistry [1]. That said, the formulation of these problems is wellposed by a system of partial differential equations and algebraic constraints that represent the conservation of mass and energy, as well as considerations of momentum and individual species while being subjected to convection, diffusion, and chemical reaction. All these phenomena are captured in direct numerical simulations with appropriate boundary conditions, but their solutions, due to these equations being highly nonlinear and strongly coupled, come at a significant computational cost [2], and thereby create the need for model-level understanding.

The focus of this paper is on premixed combustion in the Damköhler and Karlovitz regimes where reaction occurs in identifiable flame structures that are locally subjected to unsteadiness in the flow. The objective of this paper is to further develop a model of a flame for the practical purpose of capturing the impact of unsteadiness on the rates of conversion from reactants to products. To be explicit, no consideration is given here to spatial variations of composition or temperature along a flame, which can be another important characteristic of turbulent flames that are not perfectly mixed.

Important insights into the relevance of this problem can be gleaned from considering simpler nonturbulent situations of prescribed unsteady or periodic hydrodynamics to interact with a flame. Aldredge [3], [4] considered a flow field under the condition of weak flame stretch to analyze the dependency of turbulent premixed burning velocity on the intensity of flow velocity fluctuations. It was concluded that at least part of the "bending effect," which is normally seen in premixed combustion 
occurring in high-intensity turbulence [5], results from local flame stretch modifying the local consumption speed. For a better understanding of the relationship between flame surface advection and the intensity of flow excitation, Aldredge [6] studied the advection of a flame front in a transient periodic flow (spatially and temporally) where different levels of turbulent intensity of flow perturbations were considered analytically and numerically. In recent research, Aldredge [7], has studied the flame surface and burning rate increase due to wrinkling by analyzing the effect of multiscale periodic transient flow on isothermal-flame propagation. It was concluded that flames behave differently in low, intermediate and high intensities. In Aldredge's work, due to the restriction of transient flame surface growth, the local normal propagation speed was kept constant and equal to the adiabatic planar flame (laminar flame speed). These results were consistent with those obtained in earlier investigations, but also showed that flames behave differently in low, moderate, and high velocity fluctuations. It was also confirmed that the unsteady stretch rate effects and transient response of laminar flames should be considered in non-DNS turbulent modeling of flames.

It has been suggested that the transient response of stretched flames in turbulent combustion could be captured through an overall Markstein number, modified with the frequency and flame transit time [8]. This approach only seeks to estimate the overall turbulent flame speed while foregoing any information of the local and inhomogeneous rates of heat release, and it is unclear if it can address the challenges of operating across the spectrum of frequencies previously observed.

A conceptual modeling approached that allows for estimation of local burning rates with pre-calculated complex chemistry is based on flamelets, which when summed together collectively represents a turbulent flame. In their classical premixed form, flamelets provide a local consumption speed of the flame (referenced to their reactant state), which are generally assumed to be local planar structures subjected to a specified steady strain rate and of fixed reactant composition. (This consumption speed, 
$S_{L}$, is the rate mass is converted from reactants to equilibrium products per unit area of flame, and divided by the density of the reactants, and can readily be converted to the local heat release rate per unit flame area knowing the enthalpy of reaction.) The validity of these assumptions has been challenged in terms of the response of laminar flames to changing positive strain rates [9], or mixture composition fluctuations [10]. Key observations for these planar flames included that the flame responded in a quasi-steady manner at low frequencies, but at high frequencies the burning rate response became significantly attenuated, as well as having a phase lag. Clavin and Joulin showed that in high frequency, the flame behavior is universal (regardless of Lewis number) [11]. Sahafzadeh et al. [12] showed the same results occur when flame stretch was created through the motion of a curved flame where both positive and negative transient stretch rates were applied. As a result, within an unsteady flow, classical flamelet models would predict the wrong magnitude of local burning, and any expected maxima or minima events would occur at the wrong time. Lastly, an alternate approach to reduce the computational load of complex chemistry is to use reduced chemistry and simple transport properties. It has been found that the transient response of a stretched flame is not necessarily captured accurately due to the change in the internal flame structure [13].

In the current work, the approach is to model the full chemistry and then estimate the instantaneous local consumption speed by a frequency response analysis in the form of a flame transfer function using the local stretch history as the input. The broader concept using a transfer function in combustion has been used to study flame instabilities [14]. Linear [15] and non-linear [16] frequency response analyses of laminar [17] and turbulent [18] premixed flames have been considered. The motivation of the aforementioned works were to describe the flame response to flow velocity perturbations as a flame transfer function in order to study and model flame instabilities due to acoustic waves. In [19], the transfer function is defined by the ratio of the normalized flame area to velocity fluctuations. In 
theoretical studies, such as [20], a $G$-equation was used to characterize the flame front by its mean and perturbation components. Then solving by Laplace transforms the frequency response was obtained. Thermo-acoustic instabilities have been studied using a nonlinear describing function (instead of a linear flame transfer function), which was determined experimentally [16]. The flame response was estimated as a function of frequency and amplitude of perturbation acting on the combustion region. It was concluded that nonlinear mechanisms dominate the dynamics of real systems and gave a better representation of the flame dynamics. Therefore, linear and nonlinear flame transfer functions or flame describing functions have been widely used to study the flame instability in turbulent combustion. In these functions, the input was considered to be velocity fluctuations and output was usually heat release.

Although the mathematical approach is similar to that of instability analysis, in this study the frequency response analysis was obtained based on detailed numerical simulations of laminar premixed flames in order to connect stretch rate to local consumption speed in unsteady situations. Frequency response analysis provides simple closed mathematical relations that can replace large portions of the system of partial differential equations used in direct numerical simulations. Transfer functions are also independent of the form of the input excitations; therefore, the system response can be developed on one set of inputs (e.g., sinusoidal) and then used for any arbitrary inputs such as step functions, ramp functions, or the unsteadiness of turbulence.

\section{Frequency Response Analysis}

To create a useful transfer function from a frequency response analysis, consideration needs to be given to the input and output of the transfer function. Within the context of premixed turbulent flame modeling in the flamelet regime, the goal is often to represent the flame as an interface with burning 
rates that vary over the surface depending on local conditions. For example, basic flamelet libraries are used to assign a consumption speed to an element of the flame depending on its equivalence ratio and stretch rate. Since one of the goals of the current work is to better account for the unsteadiness in turbulent flames, which includes unsteadiness in stretch rate, the input to the transfer function was chosen to be the varying stretch rate and the output is the varying local consumption speed. By choosing stretch rate, the transfer function is expected to be independent of the geometry that creates it, and therefore applicable to flamelets of a turbulent flame.

The numerically modeled flame used to produce the results for this frequency response analysis has been previously reported in the combustion literature [12], and is therefore only briefly described here.

Fig. 1 shows the one-dimensional laminar premixed flame that was simulated in a cylindrically symmetric geometry. Reactants of methane and air of a specifiable equivalence ratio flow radially outward from the inlet boundary $(\mathrm{r}=5 \mathrm{~cm})$ and through the outlet boundary $(\mathrm{r}=11 \mathrm{~cm})$. The mean and fluctuating components of the inlet mass flow rate were specified so that all the chemical reactions occur within the domain and are well removed from the boundaries. The model is based on a finite volume approach for solving the discretized equations for mass, momentum, energy, and species. The thermodynamic and transport properties come from CHEMKIN, and the reaction mechanism is based on GRI 3.0 involving 36 species and 219 reactions.

For the simulations, the mass flow rate was changed sinusoidally with time (Eq. 1) at the inlet boundary.

$$
\dot{m}(t)=\dot{m}_{0}+B \sin (\omega t)
$$


In Eq. $1, \dot{m}$ is the mass flow rate at the inlet boundary, $\dot{m}_{0}$ the mean mass flow rate at the steady state condition, $B$ represents the amplitude of the oscillation, and $\omega$ is the angular frequency. The frequency was varied between 5 and $2000 \mathrm{~Hz}$. The reason to consider frequencies up to $2000 \mathrm{~Hz}$ is for the relevance to turbulent flows. For example, in the middle of a premixed turbulent duct flame stabilized by a backward facing step, mean frequency of the flame motion across a fixed point was observed to be in this range [21]. In the current study, the equivalence ratio was varied from 1.0 to 0.7 in order to compare the stoichiometric condition to lean flames, which are usually of interest due to their applicability to low-emission combustion systems.

The geometry in Fig. 1 has unique features of flame dynamics relative to the counter-flow configuration, which is usually used to generate flamelet libraries. The steady flame is unstretched due to the balance of curvature and flow divergence (i.e., strain). However, when the flame is exposed to a flow field fluctuation, the expanding flame is subjected to positive stretch, while the contracting flame generates negative stretch, which has generally been ignored in the literature. The magnitude of stretch rate, shown in Eq. 2 for an interface, could be changed by varying mean flame curvature, amplitude and frequency of oscillations, equivalence ratio, and fuel type.

$$
\kappa=\frac{1}{A^{*}} \frac{d A^{*}}{d t}=\frac{1}{r^{*}} \frac{d r^{*}}{d t}
$$

where $\kappa$ is the stretch rate, $A^{*}$ is the representative flame area, $r^{*}$ is the representative flame radius, and $t$ is time. The choice of the representative marker, usually associated with a scalar field, to convert a flame with internal structure spread over space to an interface affects the magnitude of the stretch rate. To reduce this sensitivity, it is common to choose a marker that is on the trailing edge of the flame structure [22]. The flame marker used in this work is an isotherm based on the temperature field with 
a value of $90 \%$ of the maximum temperature change. Once calculated, the changing stretch rate become the input for the response analysis to create the transfer function.

The output for the response analysis is related to the total heat release, which is the chemical energy source term integrated for all species and over all control volumes in the domain, and is defined by Eq. 3.

$$
\dot{E}_{b}=\sum_{i=1}^{N_{c v}} \sum_{k=1}^{N_{s p}} h_{k} \dot{\omega}_{k} \mathbb{V}(i)
$$

In Eq. $3, \dot{E}_{b}$ is referred to here as the rate of conversion from chemical enthalpy to sensible enthalpy for the whole flame, otherwise known as the burning rate, $\mathbb{V}(i)$ represents the volume of cell $i, h_{k}$ is the molar specific enthalpy of species $k$, and $\dot{\omega}_{k}$ is the molar rate of creation of species $k$ per unit volume. Two global characteristics of the burning rate (i.e., amplitude attenuation and phase angle change) can be observed in Fig. 2 where the burning rate has been normalized by the burning rate for the steady flame at the average mass flow rate. The solid line in all figures illustrates the quasi-steady response of the flame to a change in inlet mass flow rate. In the quasi-steady condition, the flame responds to the inlet mass flow rate instantaneously regardless of the equivalence ratio and the frequency of the oscillation, and hence the same in all the sub-figures in Fig. 2. However, the transient response depends on the equivalence ratio and the frequency of the perturbations. As can be seen at each frequency, the flame response is more delayed with decreasing equivalence ratio. In this condition, the amplitude of the flame response is also damped at lower equivalence ratios. At constant equivalence ratio, increasing the frequency has a similar influence on the flame response. Therefore, the phase lag increases and the amplitude decreases with an increase in frequency. 
Joulin [23] illustrated that the differential diffusion effect of heat and deficient reactants becomes more trivial when the frequency of oscillations reaches high limits. At that point, the Lewis-number-effects, or in this case the equivalence ratio influence, vanishes.

In Fig. 3, a similar analysis was performed to study the effect of the amplitude of oscillation and the mean flame curvature, but using a phase plot to show how the instantaneous normalized burning rate aligns with the instantaneous normalized mass flow rate. The system progresses in time by going around the ellipses in the counterclockwise direction. The original simulations depicted in Fig. 2 were done with mean flame curvature of $8 \mathrm{~cm}$. As can be seen in Fig. 3, when the mean curvature was halved to $4 \mathrm{~cm}$, the transient results were not changed significantly.

It was concluded from the two cases presented in Fig. 3 that the ellipses, illustrating the transient responses, do not rotate (i.e., do not indicate phase lag) when changing the mean flame curvature or the amplitudes. This behavior can be explained by the fact that neither the chemistry nor the fluid flow time scales are changed between the cases. In general, as discussed in [12], an important dimensionless number for controlling the transient response is the Damköhler number, which includes both flow and chemistry time scales.

A useful illustration of transient response of a flame is to plot the stretch rate versus the total burning rate. As shown in Fig. 4, the quasi-steady response is a vertical line, which indicates that the flame is unstretched at each instant. In this regard, the stretch rate at each time is zero, therefore, the energy release has sufficient time to keep up with the mass flow rate change (i.e., flame area changes) and illustrates the steady state values. Considering the transient response at $20 \mathrm{~Hz}$, a vertical narrow ellipse appears. At this condition, the range of stretch rate is small; however, the deviation from the quasisteady line can be easily observed. As the frequency is increased, the ellipses rotate about the center point and the range of stretch rate increases for the same amplitude in mass flow rate. At high 
frequencies, the amplitude in burning rate fluctuations become small as there is limited time for the flame structure to respond.

A problem with Fig. 4 is that the burning rate captures both the changing structure of the flame, which affects the rate of converting reactant to products, and the changing size of the flame. To separate these two effects, the burning rate was converted to a consumption speed by associating the burning rate with the same interface marker used to estimate the stretch rate, as well as the enthalpy of reaction and density of the reactants to create this speed. Fig. 5 shows this instantaneous consumption speed as a phase plot with respect to the corresponding instantaneous stretch rate. The quasi-steady response is a point (not shown in the figure) located on $\kappa=0$ and $S_{L}=0.286 \mathrm{~m} / \mathrm{s}$, which is essentially the steady state value and would be referred to as $S_{L}^{O}$. At very low frequencies (e.g., $20 \mathrm{~Hz}$ ), the relationship between local flame speed and stretch rate is almost linear. By further increasing the frequency, the curve becomes an ellipse. The slopes of the ellipses are negative which has been shown in other works for methane-air flames in the same range of equivalence ratio [1]. The response of the local flame speed to the stretch rate first increases (i.e., the vertical amplitude of the ellipse) in the low frequency regime and then decreases in the high frequency regime. For the case of $\varphi=0.8$, this changing behavior is seen in going from 100 to $200 \mathrm{~Hz}$ at which frequencies the consumption speed increases, to 200 to $500 \mathrm{~Hz}$ at which frequencies the consumption speed decreases. Hence, there seems to be separate low and high frequency responses.

From this analysis, it is obvious that the flame response to a changing stretch rate depends on parameters such as equivalence ratio and the frequency of oscillations. The effect of equivalence ratio can be compared to Aldredge's work [24] where he has determined Markstein numbers for lean and rich methane-air mixtures. His work, which describes the response of a thin laminar flame to acoustic flow field excitation, indicates that the influence of the stretch rate on the flame speed is decreased 
from stoichiometric conditions to lean mixtures. The same behavior has been observed in this study for a methane-air flame with equivalence ratio range of unity down to 0.7 . Chen and $\operatorname{Im}[25]$ performed a direct numerical simulation on a premixed turbulent methane-air flame which revealed that the flame speed response becomes less sensitive to turbulent flows for higher turbulence intensities. The results shown in Fig. 5 clearly illustrate a decrease in flame speed sensitivity to higher stretch rate with increasing frequency. These studies indicate that the unsteady response of a premixed flame to flow

perturbations is an important parameter in modifying turbulent flame modeling. Therefore, it would seem desirable to find a describing function to replace the cumbersome complex chemistry computations of transient response of turbulent and laminar premixed flames.

In the remainder of this section the capabilities of different models have been studied in order to estimate the transient responses in terms of the consumption speed, and these predicted values were then compared to those of full complex chemistry numerical results. The models chosen for this analysis include treatment of the system as linear first order, higher order linear, and a specific type of non-linear system. It is worth noting that this analysis only includes mass flow amplitudes of $10 \%$, which created modest fluctuating stretch rates (approximately $+/-300 \mathrm{~s}^{-1}$ for $\varphi=0.8$ ).

\subsection{First Order System Analysis}

In this subsection, a first order linear model was used to analyze the flame behavior at each equivalence ratio. Equation 4 indicates the generalized relationship between different parameters of a first order linear system.

$$
\frac{B}{A}=\frac{k}{\sqrt{\tau^{2} \omega^{2}+1}}
$$


In this equation, $\boldsymbol{B}$ is the amplitude of the output, $\boldsymbol{A}$ is the amplitude of the input, $\boldsymbol{k}$ is the gain of the system, $\boldsymbol{\tau}$ is the time constant, and $\boldsymbol{\omega}$ is the angular frequency of oscillations. For our purposes, $\boldsymbol{B}$ is the amplitude of $S_{L}$ normalized by the unstretched laminar flame speed, while $\boldsymbol{A}$ is the amplitude of stretch rate $(\kappa)$. This ratio of consumption speed to stretch rate should not be interpreted as a classical Markstein number as the extrema of the two fluctuating quantities occur at different times. Figure 6a shows the linear first order model fitted to the numerical results for an equivalence ratio of 1.0 over the entire range of frequencies. Fig. 6c shows analogous results for an equivalence ratio of 0.7. Given the previous discussion with respect to Fig. 5, and the differences between low and high frequencies, the first order linear model does a poor job in capturing the response across a wide range of frequencies. As defined in [12], in this context, low-frequency is the range where the chemistry time scale, which depends on the equivalence ratio, is smaller than the flow time scale (inverse of frequency of oscillation). Using data at each equivalence ratio, the linear first order approximation works well for the low frequency regime (below what is referred to as the cut-off frequency), as shown in Figs. 6a and 6c. A cut-off frequency has also been observed in the unsteady response of strained diffusion flames [26]. In [26], the cut-off frequency of a non-premixed flame in a counter-flow geometry separates the quasi-steady and transient regimes.

In an attempt to improve this model over the entire frequency range, the next natural step is to consider a non-linear model. Adding a nonlinearity parameter $(\xi)$ to the denominator in Eq. 4 results in:

$$
\frac{B}{A}=\frac{k}{\left(\tau^{2} \omega^{2}+1\right)^{\xi}}
$$

Figures $6 \mathrm{~b}$ and $6 \mathrm{~d}$ show the potential of nonlinear models to accurately predict transient responses for equivalence ratios of 1.0 and 0.7 , respectively. While this curve fit shows good agreement over the 
whole range of frequencies, the nonlinear transfer functions cannot be used predictively with the current approach because inverse the Laplace transform of a nonlinear function that is needed to get the transfer function is not possible. Thus, an alternative methodology is used in the next section.

Table 1 shows the parameters that resulted from the best fits of the first order linear and non-linear models where different segments of the data were considered (i.e., below the cutoff frequency, above the cutoff frequency, and all frequencies). The table also quantifies the quality of the fits through a correlation coefficient $\left(r^{2}\right)$, which compares the model's time series predictions to the full numerical simulations. One important result from this table is that first order linear transfer functions can predict transient responses with over $90 \%$ accuracy in the low frequency regime at each equivalence ratio. However, in the high frequency regime, the first order linear model fails to estimate the flame response. One possible reason could be the modifications of the internal flame structure when the flow time scale approaches chemical time scale. It should be noted that by using $\xi$, the quality of the fit improves significantly such that $r^{2}$ reaches $99 \%$. Joulin [27] studied the response of premixed flames to timedependent strain and curvature, analytically. According to Joulin's work, curvature and strain Markstein numbers are integral operators with memory kernels, meaning that the value of Markstein numbers depend on the flame geometry, fuel type, and frequency of oscillation in a transient flow. In Joulin's study, the strain Markstein number approaches zero at very high frequencies (where the fluid time scale is on the order of the chemistry time scale), while the curvature effect does not vanish. Since in this study, the flame is stretched due to the moving curved flame front, similar behaviour to Joulin's analytical work can be observed qualitatively. Clavin and Joulin [11] extended their analytical work to calculate the higher order linear transfer functions for the Markstein number estimation to account for the memory effects. 


\subsection{Linear Higher Order System Analysis}

In this section, a Polynomial Discrete-Time ARX Model [28], which is a generalized transfer function, has been used. Equation 6 shows the general form of linear higher order transfer functions.

$$
C\left(z^{-1}\right) y(t)=\sum_{i=1}^{n u} D_{i}\left(z^{-1}\right) u_{i}\left(t-n k_{i}\right)
$$

In this equation, $\mathrm{u}(t)$ is the input (i.e., stretch rate), $y(t)$ is the output (consumption speed), $C$ and $D$ are polynomials expressed in the time-shift operator $z^{-1}, u_{i}$ is the $i^{t h}$ input, $n u$, the total number of inputs, and $n k_{i}$, the $i^{t h}$ input delay that characterizes the transport delay. In the present study, the system is SISO (single input/single output), therefore the transfer function takes a more familiar form wherein $i=1$. A $Z$-transform (Time-Discrete Fourier Transformation) can be used to convert the discrete-time form of the transfer function (Eq. 6) into a complex frequency domain representation.

To test the robustness of this model, the approach was to estimate the transfer function of the flame using just a low frequency datum (e.g., for $\varphi=0.8$ the model was built from the data at $20 \mathrm{~Hz}$, and then the high frequency response was predicted).

To quantify the performance of different transfer function models, the accuracy $(\aleph)$ relative to the full numerical simulation is defined as:

$$
\aleph=\left(1-\frac{\operatorname{norm}\left(S_{L}-\widehat{S}_{L}\right)}{\operatorname{norm}\left(S_{L}-\operatorname{mean}\left(S_{L}\right)\right)}\right) \times 100
$$


where $S_{L}$ is the full numerical simulation output of the system, $\widehat{S_{L}}$ the predicted output, norm of a vector $v$ with $N$ elements is a function defined as $\left[\sum_{k=1}^{N}\left|v_{k}\right|^{p}\right]^{1 / p}$ where $p$ is a positive real value, and mean returns the average of the output.

Based on Eq. 7, it was observed that the accuracy of predictions decreases with increasing frequency. Increasing error in prediction of the transient response in the low frequency regime confirms that nonlinearity of the system increases with increasing frequency at each equivalence ratio. However, it could be considered insignificant until the oscillation crossed into the high frequency regime.

In the next step, the whole range of low frequencies below the cut-off frequency was used to generate the transfer function (Eq. 6), to give

$$
\begin{aligned}
& C(z)=1+0.9623 z^{-1}+0.636 z^{-2}+0.2826 z^{-3}+0.122 z^{-4} \\
& D(z)=-0.6376 z^{-1}+0.6915 z^{-2}+0.5377 z^{-3}-0.5923 z^{-4}
\end{aligned}
$$

$C(z)$ and $D(z)$ are the expressions represented in Eq. 6. The fourth order transfer function indicates that the first four terms on each side of Eq. 6 are required to have the most accurate predictions. It should be noted that $C(z)$ and $D(z)$ are usually referred to as the poles and zeros of a transfer function. Figure 7 shows data for $\varphi=0.8$ for which the average accuracy of predictions is $88 \%$ for frequencies up to $200 \mathrm{~Hz}$ for the linear ARX-L model (where the L represents being based on data from the whole low frequency regime); however, the flame response experiences a significant drop in accuracy after $200 \mathrm{~Hz}$. At $500 \mathrm{~Hz}$, the accuracy from Eq. 7 degrades to almost $-400 \%$, and is so poor that the results are not shown for higher frequencies as the range of laminar flame speeds does not allow for comparisons for the other non-linear models developed in the next section. Also shown in Fig. 7 are 
results of an ARX model, but where the $C(z)$ and $D(z)$ functions were developed on high frequency data (shown as ARX-H in the figures).

Despite going to a higher order linear model, the conclusion remains the same that the response in consumption speed is divided in two regimes based on the cut-off frequency obtained from numerical simulations of transient response of laminar premixed flames for each equivalence ratio. Although the accuracies are in an acceptable range, there may be a considerable improvement using nonlinear models to predict the output.

\subsection{Nonlinear System Analysis}

As shown in the previous section, although linear transfer functions can achieve accurate prediction in the low frequency range of flame response to changes in stretch rate, they are not able to predict the response at higher frequencies. Therefore, to capture the nonlinearities, the flame transfer function was replaced with a flame describing function [29].

One difference between linear and nonlinear transfer functions is in the representation of the equivalent time-domain. Nonlinear systems are defined based on a sequence of transfer functions, as opposed to only one function in the linear case. Although with stronger nonlinearities, more transfer functions are required to study the behavior of the system accurately. For a wide range of nonlinear systems, it is usually sufficient to capture the dominant effects by considering the first, second, and third order transfer functions. The other difference is that even in a single input/single output nonlinear system, a transfer function is multi-variant, which increases the difficulty of defining a transfer function to relate the system response to input, interpret the output, and obtain a complete understanding of its behavior. In this regard, it could be useful to apply Nonlinear Frequency Response Analysis (NFRA) on the current transient data. 
NFRA applies a sinusoidal function as the input. However, the system cannot be considered linear around the steady state condition. In this case, nonlinear perturbations occur, and therefore, similar to the flame analyzed, the transient response can no longer be expressed with a single sinusoidal function. New frequencies such as harmonics and intermodulation frequencies are generated, meaning that the sinusoidal functions of the high frequency response data can be best approximated by a sum of two or three sinusoidal functions instead of one. The fact that the current time-series data in the high frequency regime could be approximated by the sum of two or three sinusoidal functions more accurately supports this aspect of nonlinear systems for laminar premixed flames. These perturbations can be studied by higher order frequency response functions, which is a mathematical framework, such as a Volterra series [30]. The advantage of using a higher order transfer function is that it contains information about the nonlinearities of the system. Eq. 9 shows the general form of nonlinear systems in the time domain.

$$
y(t)=\sum_{n=1}^{\infty} y_{n}(t)=\sum_{n=1}^{\infty} \int_{-\infty}^{\infty} \ldots \int_{-\infty}^{\infty} h_{n}\left(\tau_{1}, \ldots, \tau_{n}\right) \prod_{i=1}^{n} u\left(t-\tau_{i}\right) d \tau_{i}
$$

$y(t)$ is the output in the time domain. It is worth noting that the number of differential elements are hidden in the $\pi$ function. Applying Fourier series, the output can be estimated using the nonlinear transfer function in the frequency domain as:

$$
Y_{n}\left(s_{1}, \ldots, s_{n}\right)=H_{n}\left(s_{1}, \ldots, s_{n}\right) U\left(s_{1}\right) \ldots U\left(s_{n}\right)
$$

With this form of the transfer function, a value of unity for $n$ results in a first order system. Notice that both the impulse response function and the transfer function are independent of the input excitation. 
This is a highly desirable feature because it enables the determination of the system response for arbitrary inputs, as will be shown in the last section of this paper.

Although higher order linear systems and nonlinear systems have multidimensional transfer functions, their behavior is significantly different. Higher order linear systems have real multidimensional input/output that are defined with integer arguments. There are different approaches to model nonlinear systems including Volterra series models, block structured models (Wiener and Hammerstein [31]), neural network models, and nonlinear auto-regressive exogenous (NARX) models, and the nonlinear auto-regressive moving average model with exogenous inputs (NARMAX) [32].

Among these predictive models, NARX showed better results in estimating the transient response of the flame for the whole range of frequencies. NARX models (which is an extension of ARX in polynomial transfer functions) are flexible nonlinear functions that are able to capture the complex behavior in nonlinear systems such as the current data set. Eq. 11 illustrates the structure of a linear SISO ARX model:

$$
\begin{aligned}
y(t)+a_{1} y(t-1)+a_{2} y(t-2)+\cdots+a_{n a} y(t-n a) & \\
& =b_{1} u(t)+b_{2} u(t-1)+\cdots+b_{n b} u(t-n b+1)+e(t)
\end{aligned}
$$

Where $u, y$ and $e$ are the input, output, and noise, respectively. This structure implies that the output $y(t)$ in current time is predicted as a weighted sum of past output values and current and past input values. $n a$ is the number of past output terms, and $n b$ is the number of past input terms used to predict the current output. It has been shown that the surface wrinkling in turbulent flames at one location, in addition to the local velocity perturbation, depends also on the flame surface fluctuations at previous times, upstream of the flame front [33]. Therefore, an ARX model could capture this 
memory effect occurring in turbulent flames by modifying the laminar flamelet response to a change in upstream flow rate. Rewriting Eq. (11) as a product gives:

$$
\begin{aligned}
y_{p}(t)=\left(-a_{1},\right. & \left.-a_{2}, \ldots,-a_{n a}, b_{1}, b_{2}, \ldots, b_{n b}\right)(y(t-1), y(t \\
& -2), \ldots, y(t-n a), u(t), u(t-1), \ldots, u(t-n b-1))
\end{aligned}
$$

where $y(t-1), y(t-2), \ldots, y(t-n a), u(t), u(t-1), \ldots, u(t-n b-1)$ are delayed input and output variables, called 'regressors.' The coefficients vector $\left(-a_{1}, \ldots,-a_{n a}, b_{1}, b_{2}, \ldots, b_{n b}\right)$ represents the weighting applied to these regressors. The linear ARX model thus predicts the current output $y_{p}$ as a weighted sum of its regressors. The flexibility of the NARX model allows for replacing the weighted sum of the regressors in a linear model with a nonlinear functions, $F$.

$$
y_{p}(t)=F(y(t-1), y(t-2), y(t-3), \ldots, u(t), u(t-1), u(t-2), \ldots)
$$

The nonlinearity estimator $(F)$ for calculating the NARX model in this study was wavenet [34], which stores the wavelet network for use in transfer functions. Different parameters of this network are automatically estimated in the system identification toolbox of MATLAB.

The NARX model was applied to three different data sets, including the low frequency regime, the high frequency regime, and the entire range of frequencies (shown in Fig. 7 as NARX-L, NARX-H, and NARX-A, respectively, along with the ARX model for comparison). As can be seen in Fig. 7, ARX-L, NARX-A, and NARX-L have been used to predict the low frequency regime. Among these models, NARX-L is capable of producing results with accuracy over $99 \%$. However, as can be observed in the $500 \mathrm{~Hz}$ plot in Fig. 7, NARX-L fails to predict the numerical data. Thus, this model 
has been eliminated from the rest of the high frequency plots $(1000 \mathrm{~Hz}$ and $2000 \mathrm{~Hz})$. In the high frequency regime, ARX-H, NARX-A, and NARX-H are compared to the numerical results. Among these models, NARX-H shows the highest accuracy (99\%), while the NARX-A at 95\% accuracy is very good and comes with the added feature of being a single model that is quite effective over the whole range of frequencies. It should also be noted that these models (ARX and NARX) are all capable of predicting the transient responses due to storing time histories in their structures. However, because of the nonlinear relationship between the input and output at high frequencies, the nonlinear models tend to be more accurate.

\subsection{Prediction of a Random Input Response Using Transfer Functions}

Turbulent flows consist of flow perturbations that are not simple sinusoidal functions. For our purposes here, these fluctuations were modelled as a sum of different sinusoidal functions with different amplitudes, frequencies, and phase lags. The transfer functions developed in the previous sections were then used to predict the transient responses to seemingly random input. In order to test this capability, three different ranges of frequency were selected including low and high frequency ranges, and a combination of the two.

First, three frequencies are used to form the input in the low frequency regime by summing them. Frequencies within the low range were selected $(50 \mathrm{~Hz}, 80 \mathrm{~Hz}$, and $100 \mathrm{~Hz})$ which results in forming a stretch rate perturbation in Fig. 8 (top figure). This input was used to compare the responses from the transfer function (NARX-L) to the results from detailed numerical calculations with complex chemistry (Fig. 8 - bottom figure). In Fig. 8 (bottom figure), the solid line curve denotes the numerical results obtained from the code based on full complex chemistry, which was originally used to calculate the 
transient response at each individual case, and the dashed line curve shows the transfer function (NARX-L) output.

The same approach was taken to predict the response using the transfer function in the previous section for high frequency regime $(500 \mathrm{~Hz}, 1000 \mathrm{~Hz}$, and $2000 \mathrm{~Hz})$. In this condition, the deviation of quasisteady response from the complex chemistry transient simulation is more significant compared to low frequency analysis. As depicted in Fig. 9, the transfer function (NARX-H) is capable of predicting the transient response with accuracy of $83 \%$.

The final test was to consider a combination of low and high frequencies $(80 \mathrm{~Hz}, 100 \mathrm{~Hz}, 200 \mathrm{~Hz}, 500$ Hz). As can be observed from Fig. 10, the transfer function (NARX-A) is capable of producing the transient results with $63 \%$ accuracy.

Comparing the three cases explained above, it is clear that low and high frequency regimes can be predicted accurately using a transfer function generated for each separate regime. Although the combined frequency range does not show high accuracy compared to the other cases, it shows considerable improvement compared to modeling the flame with a fixed burning rate, which would have an accuracy of $0 \%$ according to Eq. (7). It is worth mentioning that since the flamelets in a turbulent flame are generally considered time-dependent one-dimensional structures, the transfer functions, in this study, are developed for a flame that moves in the radial direction, which means that only changes in one spatial coordinate are considered in the flame structure. In a more realistic case, a flame has a 3-D structure that changes not only in the radial direction but also in angular and longitudinal coordinates. For instance, in a turbulent flame, temperature changes along the flame front due to the oscillation of stretch rate. This variable temperature could result from the flame geometry, equivalence ratio variation (fuel rich and fuel lean sites in the flame structure), etc. Therefore, as long as the temperature changes along the flame front result from the stretch oscillations, the transfer 
functions developed in this study are able to capture the flame's response. In order to have a more comprehensive model including different chemistry (fuel rich or other fuels such as hydrogen and heavier hydrocarbons), the coefficients in the transfer functions should be modified.

\section{Conclusions}

In summary, unsteady laminar premixed and turbulent flames are complicated systems underpinned by coupled heat and mass transfer, and chemical conversion processes. In this paper, an attempt was made to reduce this complexity by seeking a connection, through a transfer function, between an imposed hydrodynamic history (i.e., a varying stretch rate) and the responding rate of conversion of chemical to sensible enthalpy (i.e., the local and instantaneous consumption speed). Data for development of various transfer functions was generated by a numerical model of an inwardly burning laminar premixed lean methane/air flame in a cylindrically-symmetric geometry for different equivalence ratios $(0.7-1.0)$. The model considers cyclic exposure to positive and negative stretch rates at different frequencies $(5-2000 \mathrm{~Hz})$ as a means to embed unsteadiness and complex chemical affects into a laminar flamelet modeling approach of premixed turbulent combustion. The transfer functions considered were first-order linear, first-order nonlinear, higher order linear, and higher order nonlinear systems.

A linear first order model was applied to the data and it fit the low frequency results for each flame. However, the flame behavior deviates from that of a linear first order system as the frequency is increased approaching conditions at which the chemical and flow time scales were the same. Applying higher order linear transfer functions improved the prediction due to the capability to store the time histories, but behaviour at low and high frequencies could still not be captured through a single function. A key observation into the data supporting transfer model development was that there are 
two different response regimes. At lower frequencies, as the range of stretch rate increased, the range of consumption speeds increased, while at higher frequencies, the range of range of consumption speeds observed decreased as the range of stretch rate increased. The cutoff between these low and high frequency response behaviours was dependent on the equivalence ratio, and was associated with the ratio of chemical to hydrodynamic time of unity.

Nonlinear models, such as the nonlinear ARX model, could correctly predict the transient local flame speed over the whole range of frequencies and changing flow conditions. This transfer function could also reconstruct the local flame speed response for any arbitrary flow input, which enables the model to capture the transient effects of a flame at any conditions. Therefore, estimating a flame transfer function of laminar premixed flames could yield a better understanding of flame dynamics and could be used in order to modify the quasi-steady assumption in laminar flamelet models of turbulent combustion.

\section{Acknowledgements}

The authors would like to thank the Natural Sciences and Engineering Research Council of Canada (NSERC) for financial support. This research was undertaken, in part, thanks to funding from the Canada Research Chairs program.

\section{References}

[1] J. Driscoll, "Turbulent Premixed Combustion: Flamelet Structure and its Effect on Turbulent Burning Velocities," Prog. Energy Combust. Sci., vol. 34, no. 1, pp. 91-134, 2008.

[2] H. Wang, E. R. Hawkes, and J. H. Chen "Turbulence-flame Interactions in DNS of a Laboratory High Karlovitz Premixed Turbulent Jet Flame," Physics of Fluids, vol. 28, no. 9, 2016.

[3] R.C. Aldredge, "The Propagation of Wrinkled Premixed Flames in Spatially Periodic Shear Flow," Combustion and Flame, vol. 90, pp. 121-133, 1992.

[4] R.C. Aldredge, "The Speed of Isothermal-Front Propagation in Isotropic, Weakly Turbulent Flows," Combustion Science and Technology, vol. 178, p. 1201-1215, 2006. 
[5] G. Nivarti, S. Cant, "Direct Numerical Simulation of the Bending Effect in Turbulent Premixed Flames," Proceedings of the Combustion Institute, vol. 36, no. 2, p. 1903-1910, 2017.

[6] R.C. Aldredge, "Flame-Surface Advection in Transient Periodic Flow," Combustion Science and Technology, vol. 187, p. 148-161, 2015.

[7] R. Aldredge, "Flame Propagation in Multiscale Transient Periodic Flow," Combustion and Flame, vol. 183, p. 166-180, 2017.

[8] F. Zhang, T. Zirwes, P. Habisreuther, H. Bockhorn, "Effect of Unsteady Stretching on the Flame Local Dynamics," Combustion and Flame, vol. 175, p. 170-179, 2017.

[9] C.A. Ghoniem, A.F. Petrov, "The Transient Response of Strained Laminar-Premixed Flames," Combust. Flame, vol. 102, pp. 401-417, 1995.

[10] R. Lauvergne and F. Egolfopoulos, "Unsteady Response of $\mathrm{C}_{3} \mathrm{H}_{8} /$ Air Laminar Premixed Flames Submitted to Mixture Composition Oscillations," Proceedings of the Combustion Institute, vol. 28, p. 1841-1850, 2000.

[11] P. Clavin, G. Joulin, "High-frequency response of premixed flames to weak stretch and curvature: a variable-density analysis," Combust. Theory Modelling, vol. 1, p. 429-446, 1997.

[12] M. Sahafzadeh, L. W. Kostiuk, S. B. Dworkin "Transient Response of a Laminar Premixed Flame to a Radially Diverging/Converging Flow," Combustion and Flame, vol. 179, p. 51-62, 2017.

[13] T. Poinsot, D. Veynante, Theoretical and Numerical Combustion, Philadelphia: Edwards Inc., 2005.

[14] T. Schuller, S. Ducruix, D. Durox, and S. Candel, "Modeling Tools for the Prediction of Premixed Flame Transfer Functions," Proceedings of the Combustion Institute, vol. 29, p. 107113, 2002.

[15] H. Y. Wang, C. K. Law, T. Lieuwen, "Linear Response of Stretch-Affected Premixed Flames to Flow Oscillations," Combustion and Flame, vol. 156, p. 889-895, 2009.

[16] P. Palies, D. Durox, T. Schuller, S. Candel, "Nonlinear Combustion Instability Analysis Based on the Flame Describing Function Applied to Turbulent Premixed Swirling Flames," Combustion and Flame, vol. 158, p. 1980-1991, 2011.

[17] T. Schuller, D. Durox, S. Candel, "A Unified Model for the Prediction of Laminar Flame Transfer Functions: Comparisons Between Conical and V-Flame Dynamics," Combustion and Flame, vol. 134, p. 21-34, 2003.

[18] R. Balachandran, B. O. Ayoola, C. F. Kaminski, A. P. Dowling, E. Mastorakos, "Experimental Investigation of the Nonlinear Response of Turbulent Premixed Flames to Imposed Inlet Velocity Oscillations," Combustion and Flame, vol. 143, p. 37-55, 2005.

[19] T. Lieuwen, "Nonlinear Kinematic Response of Premixed Flames to Harmonic Velocity Disturbances," Proceedings of the Combustion Institute, vol. 30 , p. 1725-1732, 2005.

[20] S. Ducruix, D. Durox, S. Candel, "Theoretical and Experimental Determinations of the Transfer Function of a Laminar Premixed Flame," Proceedings of the Combustion Institute, vol. 28, p. 765-773, 2000.

[21] I. G. Shepherd, J. B. Moss, "Characteristic Scales for Density Fluctuations in a Turbulent Premixed Flame," Combustion Science and Technology, vol. 33, 5-6, 231-243, 1983. 
[22] G .K. Giannakopoulos, M. Matalon, C. E. Frouzakis, A. G. Tomboulides, "The Curvature Markstein Length and the Definition of Flame Displacement Speed for Stationary Spherical Flames," Proceedings of the combustion institute, vol. 35, 737-743, 2015.

[23] G. Joulin, "On The Response of Premixed Flames to Time Dependent Stretch and Curvature," Combust. Sci. and Tech., vol. 97, 219-229, 1994.

[24] R. C. Aldredge, "Methane-air Markstein Numbers from Measurements of Thermoacoustic Instability," Combust. Sci. and Tech., vol. 177, 1023-1047, 2005.

[25] J. H. Chen, H. G. Im, "Correlation of Flame Speed with Stretch in Turbulent Premixed Methane/Air Flames," Proc. Combust. Inst., vol. 27, 819-826, 1998.

[26] F.N. Egolfopoulos, C.S. Campbell, "Unsteady Counterflowing Strained Diffusion Flames: Diffusion-limited Frequency Response," J. Fluid Mech., vol. 318, 1-29, 1996.

[27] G. Joulin, "On The Response of Premixed Flames to Time-Dependent Stretch and Curvature," Combust. Sci. and Tech., vol. 97, 219-229, 1994.

[28] R. Vidal, "Recursive Identification of Switched ARX Systems," Automatica, vol. 44, no. 9, pp. 2274-2287, 2008.

[29] N. Noiray, D. Durox, T. Schuller, S. Candel, "A Unified Framework for Nonlinear Combustion Instability Analysis Based on the Flame Describing Function," J. Fluid Mech. , vol. 615, p. 139-167, 2008.

[30] M. Schetzen, The Volterra and Wiener Theories of Nonlinear Systems, Krieger Publishing Company, 2006.

[31] A. Wills, T. B. Schön, L. Ljung, B. Ninness, "Identification of Hammerstein-Wiener Models," Automatica, vol. 49, no. 1, pp. 70-81, 2013.

[32] A. Rahrooh, S. Shepard, "Identification of Nonlinear Systems Using NARMAX Model," Nonlinear Analysis: Theory, Methods \& Applications, vol. 71, no. 12, pp. e1198-e1202, 2009.

[33] S. Hemchandra, T. Lieuwen, "Local Consumption Speed of Turbulent Premixed Flames - An Analysis of "Memory Effects"," Combustion and Flame, vol. 157, no. 5, p. 955-965, 2010.

[34] Q. Zhang, "Using Wavelet Network In Nonparametric Estimation," IEEE Trans. Neural Networks, vol. 8, p. 227-236, 1997. 
Table 1. Comparison of linear model prediction accuracy and deviation from linear first order model for three different equivalence ratios of $0.7,0.8$ and 1.0 .

\begin{tabular}{|c|c|c|c|c|c|c|c|c|c|}
\hline & \multicolumn{3}{|c|}{$\varphi=0.7$} & \multicolumn{3}{|c|}{$\varphi=0.8$} & \multicolumn{3}{|c|}{$\varphi=1.0$} \\
\hline & $\begin{array}{c}\text { Lower } \\
\text { than } \\
20 \mathrm{~Hz}\end{array}$ & $\begin{array}{c}\text { Higher } \\
\text { than } \\
20 \mathrm{~Hz}\end{array}$ & $\begin{array}{c}\text { All } \\
\text { Frequencies }\end{array}$ & $\begin{array}{c}\text { Lower } \\
\text { than } \\
200 \\
\text { Hz }\end{array}$ & $\begin{array}{c}\text { Higher } \\
\text { than } \\
200 \mathrm{~Hz}\end{array}$ & $\begin{array}{c}\text { All } \\
\text { Frequencies }\end{array}$ & $\begin{array}{c}\text { Lower } \\
\text { than } \\
750 \mathrm{~Hz}\end{array}$ & $\begin{array}{c}\text { Higher } \\
\text { than } \\
750 \mathrm{~Hz}\end{array}$ & $\begin{array}{c}\text { All } \\
\text { Frequencies }\end{array}$ \\
\hline \multicolumn{10}{|c|}{ Linear First Order } \\
\hline$\tau$ & 0.0061 & 0.0065 & 0.0069 & 0.0023 & 0.0034 & 0.0047 & 0.0018 & 0.0024 & 0.0032 \\
\hline k & 0.0011 & 0.0015 & 0.0018 & 0.0008 & 0.0009 & 0.0010 & 0.0005 & 0.0006 & 0.0007 \\
\hline$r^{2}$ & 0.94 & 0.70 & 0.87 & 0.99 & 0.73 & 0.90 & 0.98 & 0.68 & 0.88 \\
\hline \multicolumn{10}{|c|}{ Non-Linear } \\
\hline $\bar{\tau}$ & 0.0053 & 0.0066 & 0.0073 & 0.0019 & 0.0027 & 0.0031 & 0.0009 & 0.0011 & 0.0017 \\
\hline k & 0.0012 & 0.0013 & 0.0014 & 0.0008 & 0.0008 & 0.0009 & 0.0005 & 0.0006 & 0.0005 \\
\hline$\xi$ & 0.35 & 0.35 & 0.35 & 0.30 & 0.30 & 0.30 & 0.25 & 0.25 & 0.25 \\
\hline$r^{2}$ & \multicolumn{9}{|c|}{$\sim 0.99$} \\
\hline
\end{tabular}




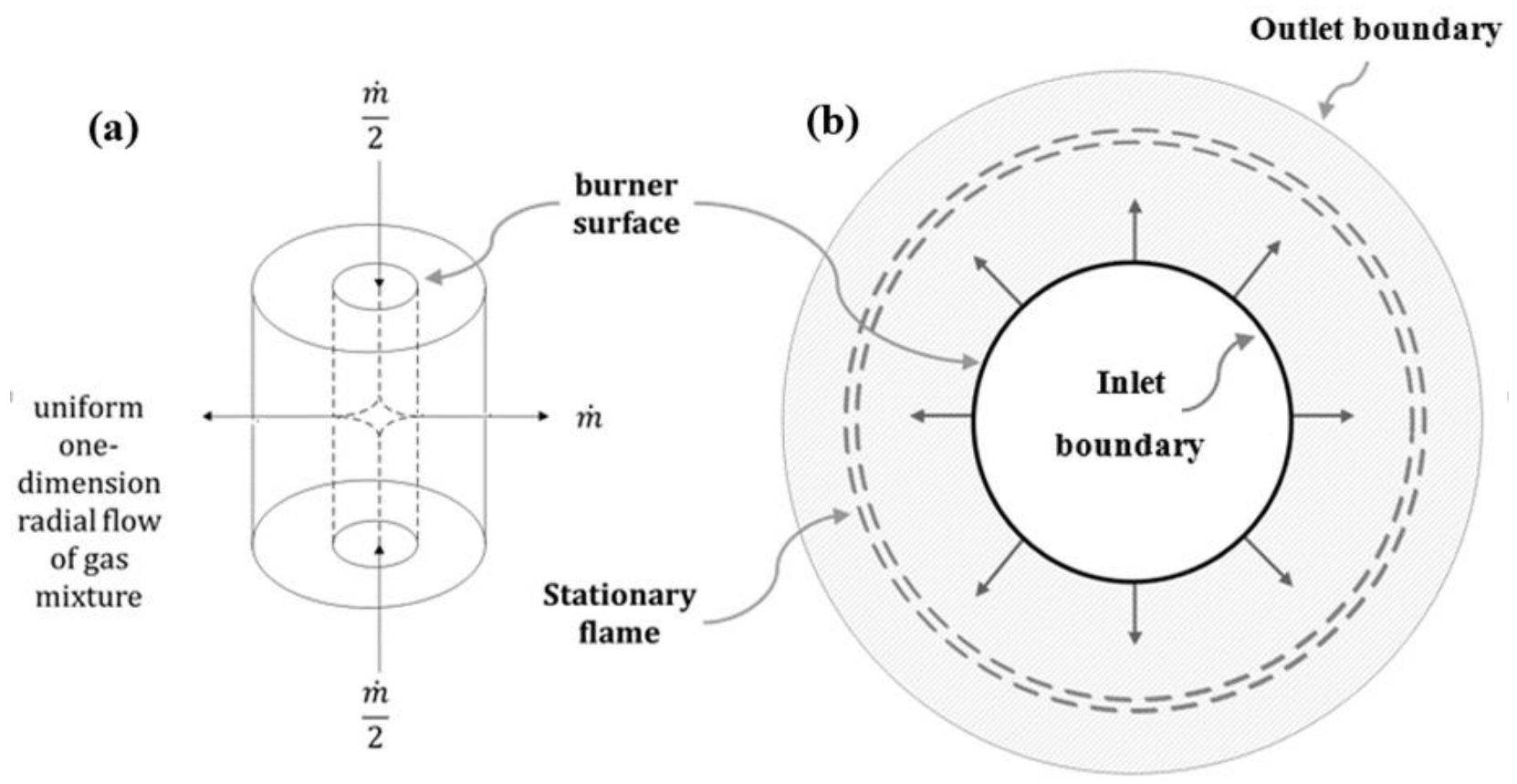

Fig. 1. (a) The cylindrically-symmetric geometry (side view), (b) Stabilized stationary flame around burner (top view) 

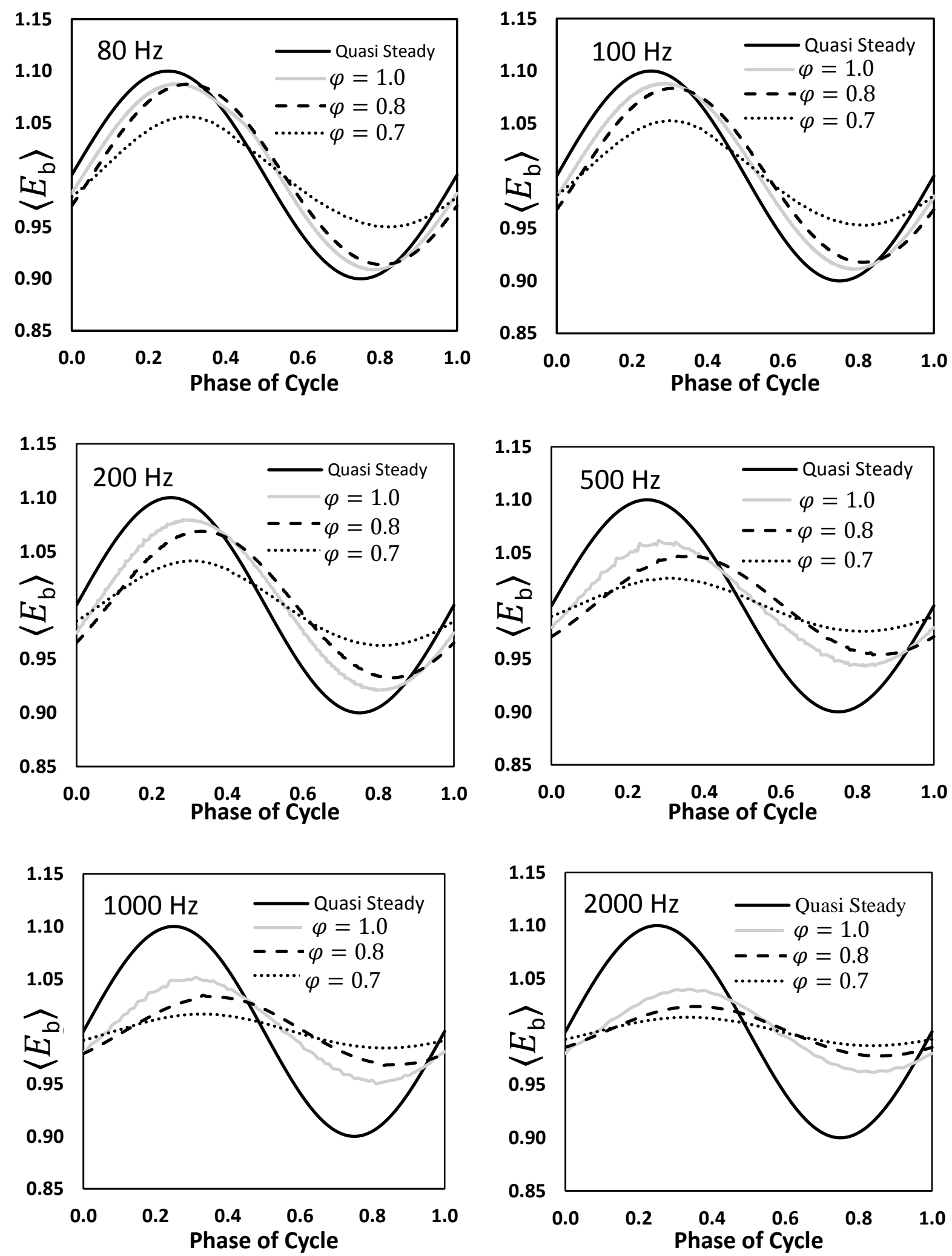

Fig. 2. Quasi steady and transient responses of flames with equivalence ratios of $\varphi=1.0, \varphi=0.8$ and $\varphi=0.7$ at a range of frequencies from $80 \mathrm{~Hz}$ to $2000 \mathrm{~Hz}$ 

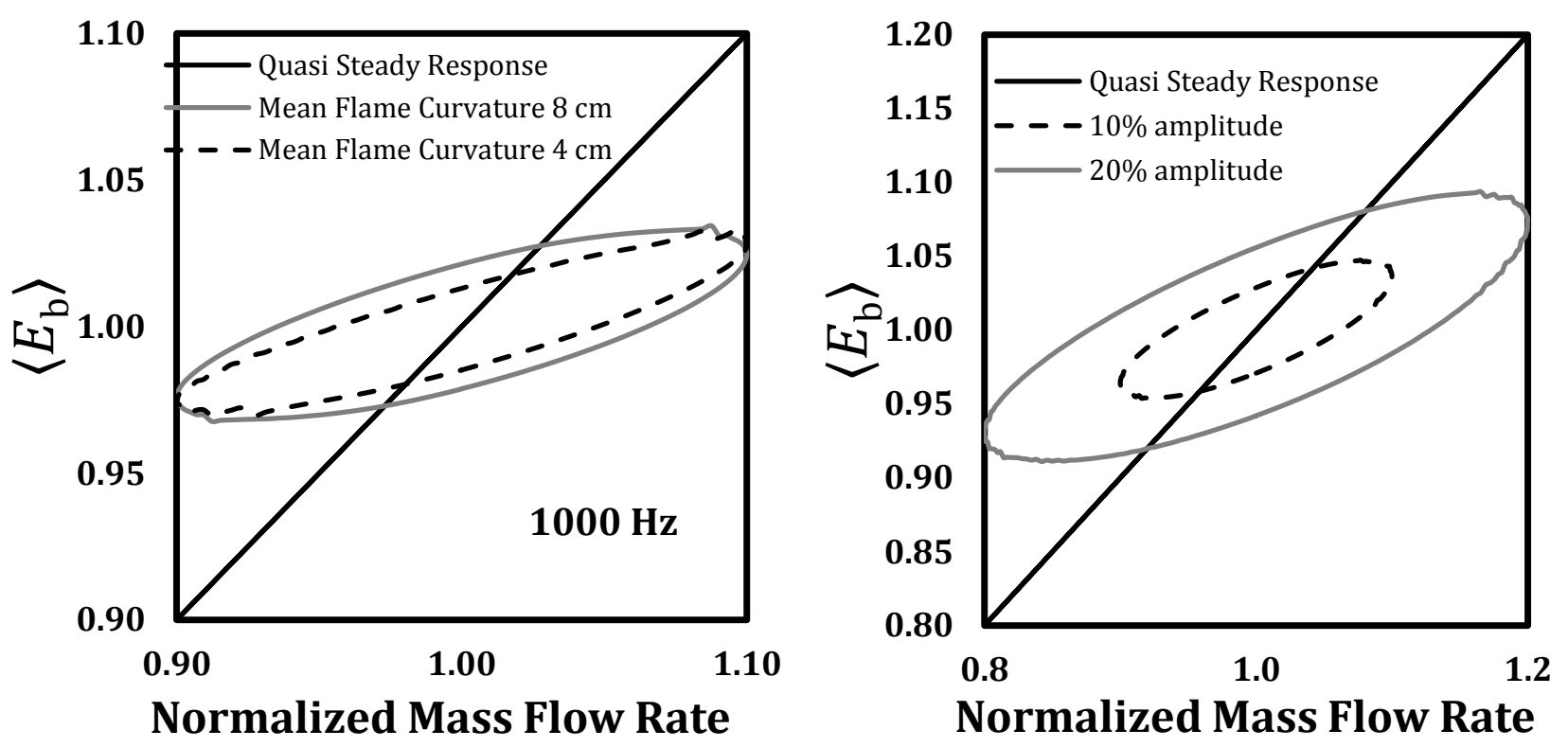

Fig. 3. Normalized $\dot{E}_{b}$ vs. normalized mass flow rate for a flame with equivalence ratio $\varphi=0.8$ and frequency $1000 \mathrm{~Hz}$; right side: amplitude of mass flow being either of $10 \%$ and $20 \%$ of the mean flow rate, left side: the results for two mean flame curvatures.

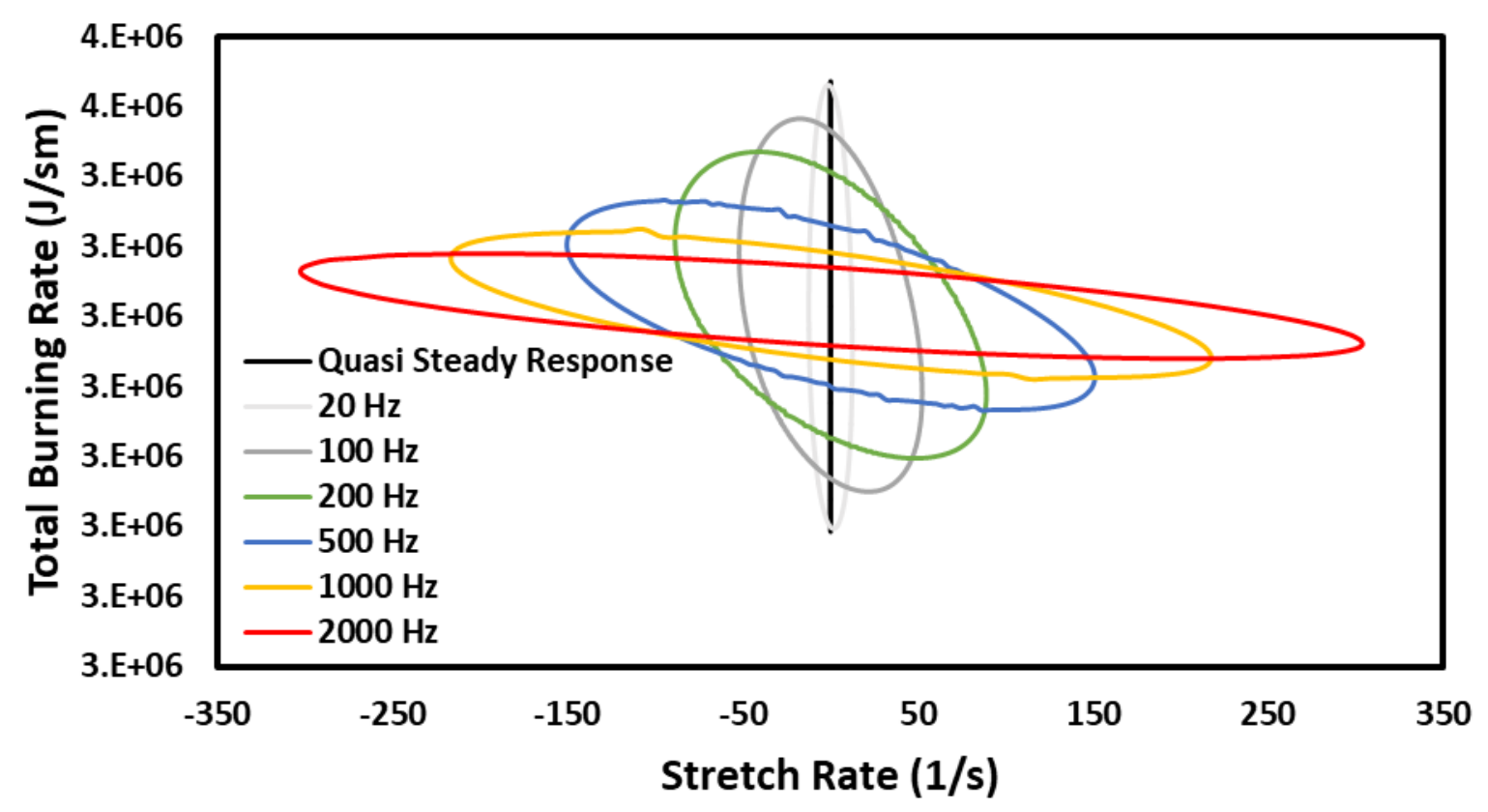

Fig. 4. Total burning rate $\left(\dot{E}_{b}\right)$ versus stretch rate for equivalence ratio $\varphi=0.8$ for quasi-steady response and frequencies from $20 \mathrm{~Hz}$ to $2000 \mathrm{~Hz}$ 


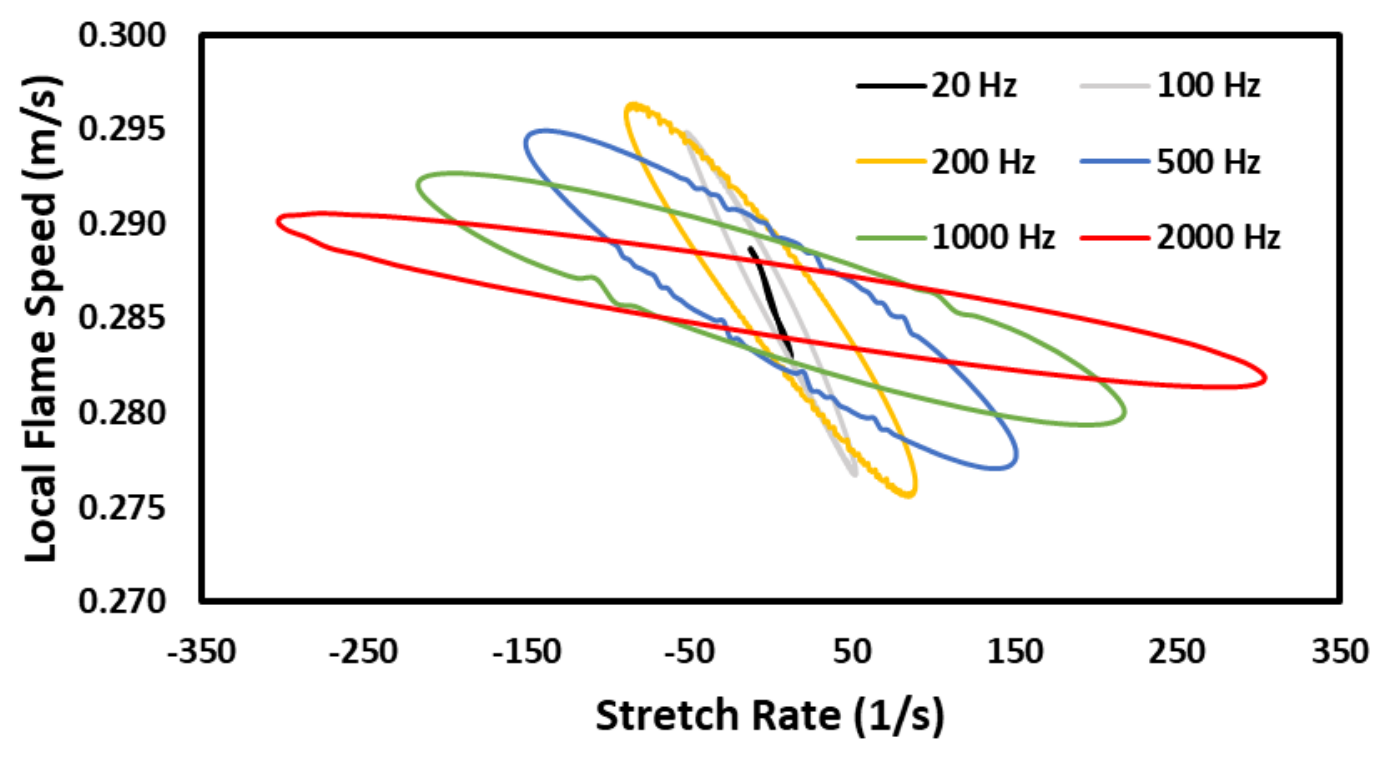

Fig. 5. Local flame speed versus stretch rate for the equivalence ratio of $\varphi=0.8$ and quasi-steady response and the frequencies between $20 \mathrm{~Hz}$ and $2000 \mathrm{~Hz}$ 

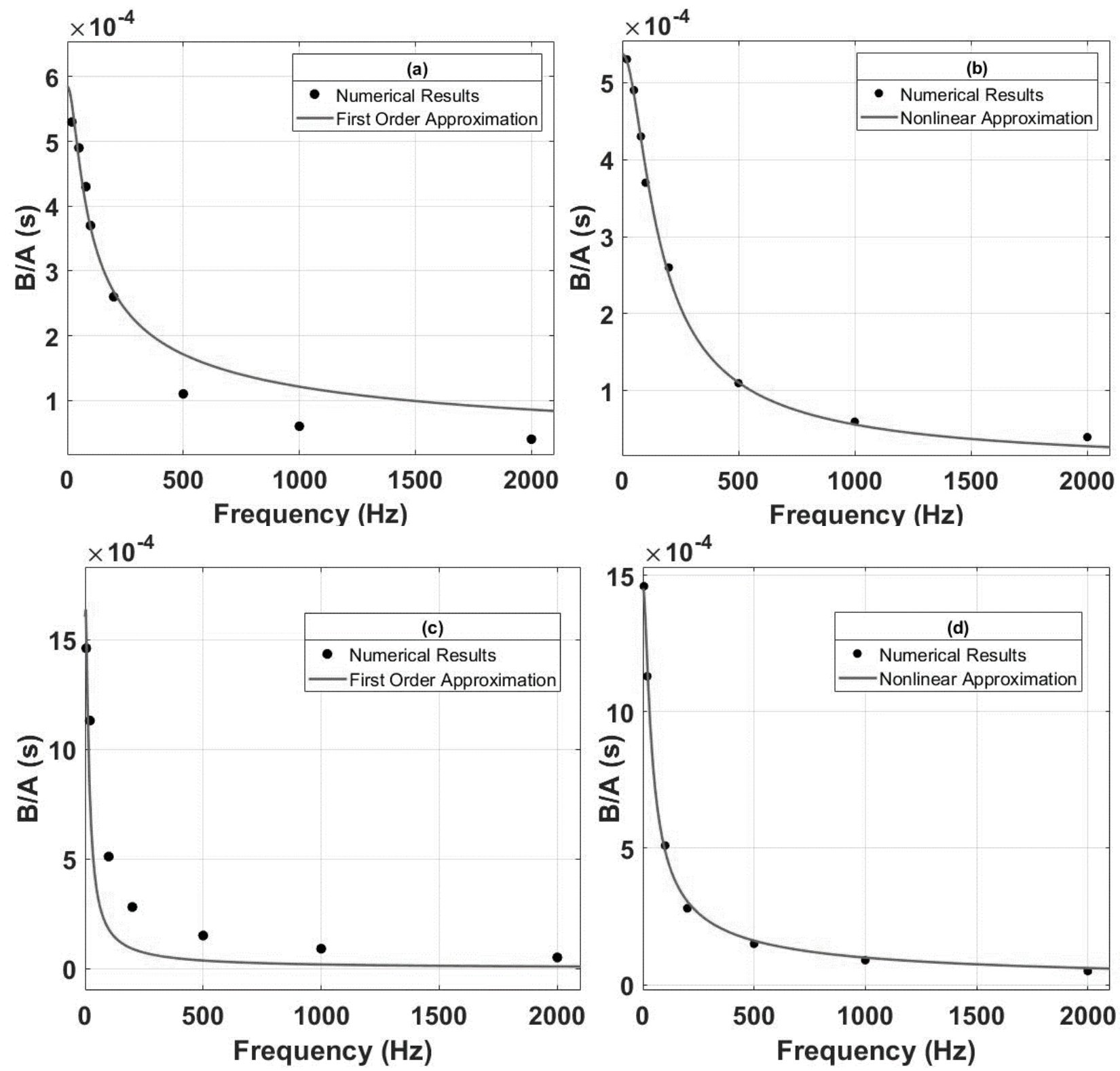

Fig. 6. Predicting transient response for the whole range of frequencies (a) Linear first order approximation for $\varphi=1.0$, (b) Nonlinear approximation for $\varphi=1.0$, (c) Linear first order approximation for $\varphi=0.7$, (d) Nonlinear approximation for $\varphi=0.7$ 

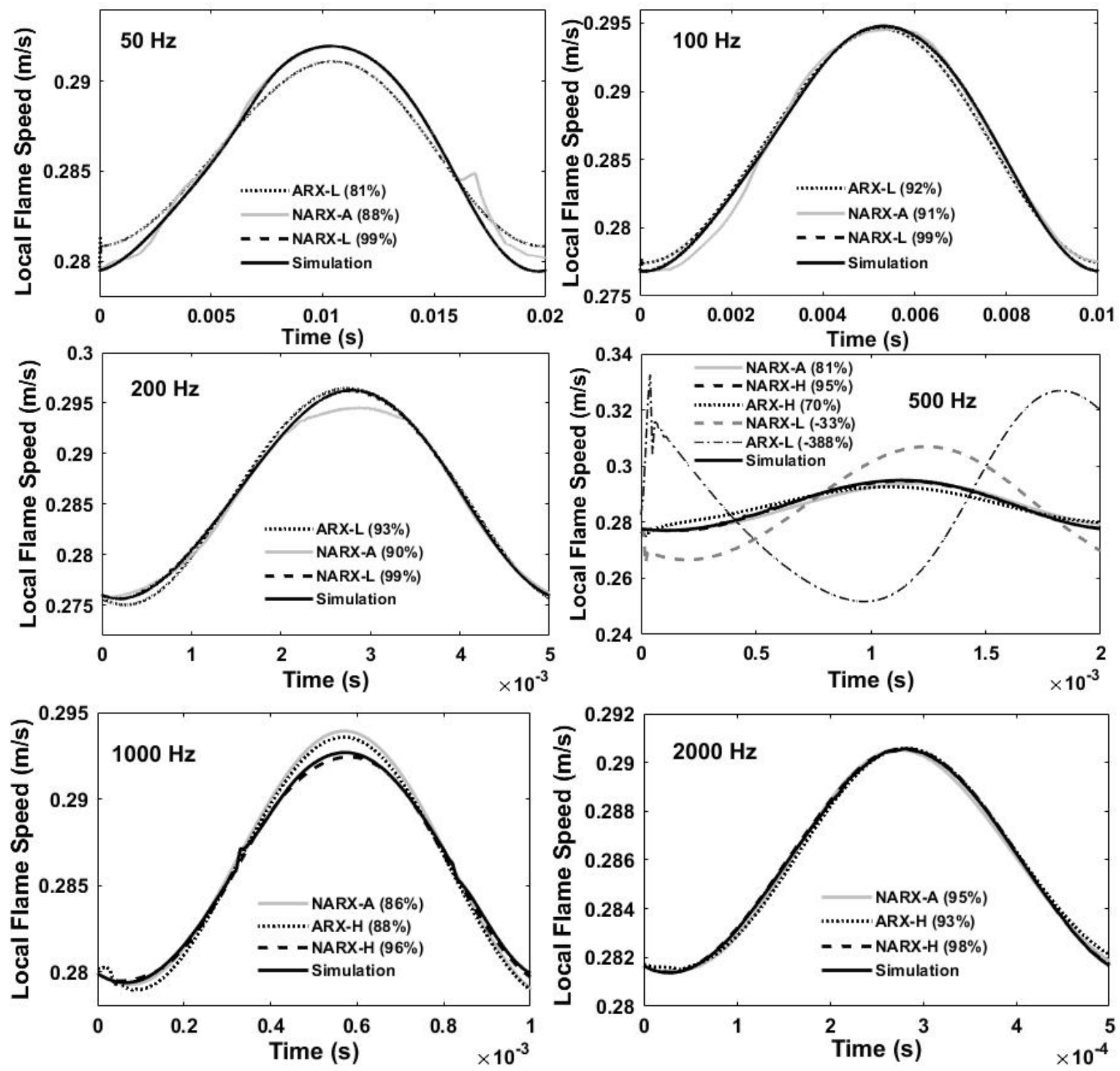

Fig. 7. Estimation of transient response of a laminar premixed flame for $\varphi=0.8$ using the higher order linear ARX model and nonlinear ARX (NARX) model. The -L, -H, and -A denote models based on data in the low frequency, high frequency, and entire frequency ranges, respectively. 

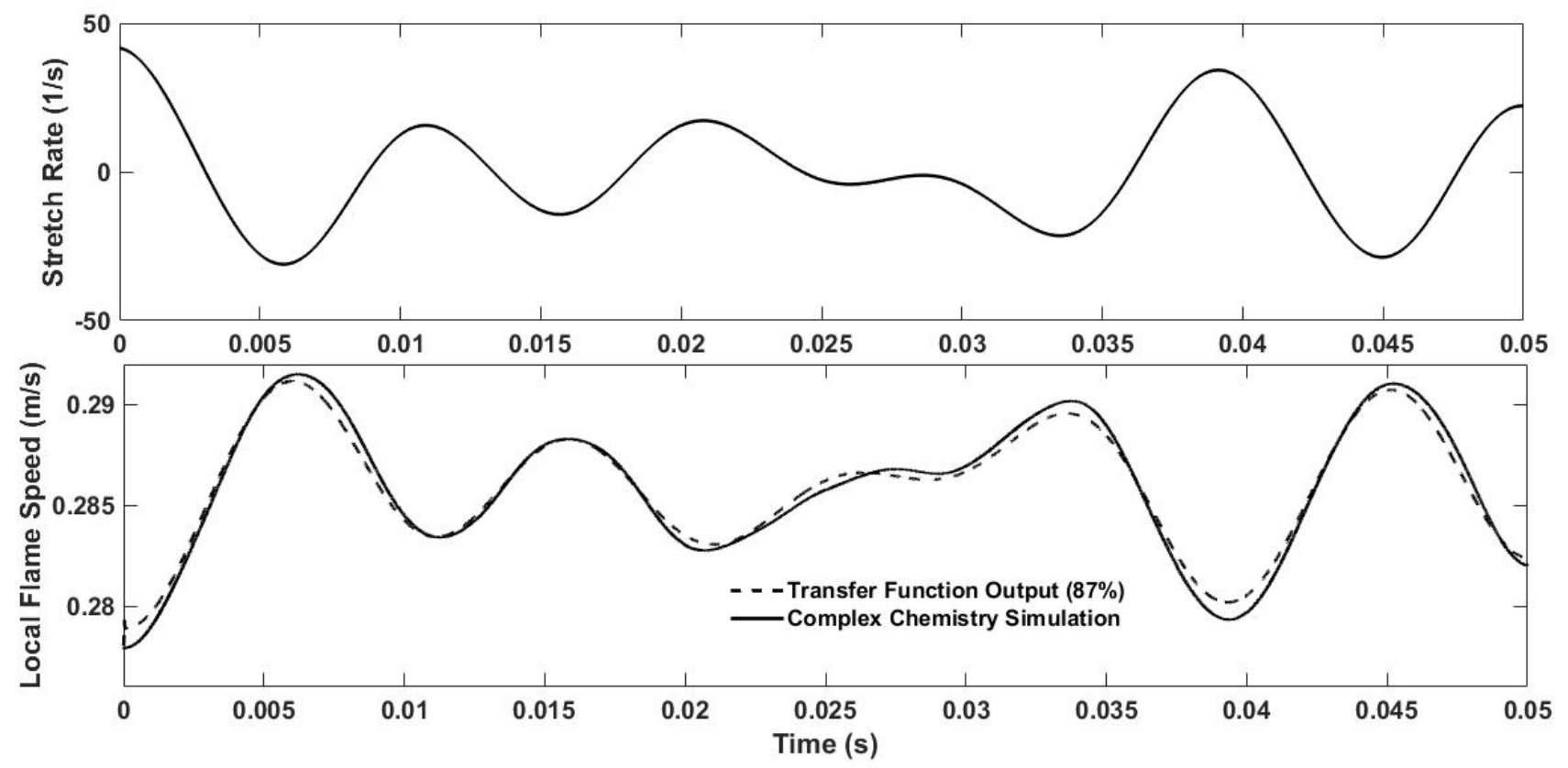

Fig. 8. Comparison of the transient response (complex chemistry) and the transfer function prediction of a laminar premixed flame to a random input in the low frequency range $(\varphi=0.8)$, Top figure: stretch rate perturbation as the input, Bottom figure: solid line - the transient response (complex chemistry) and dashed line - transfer function output (NARX-L). 

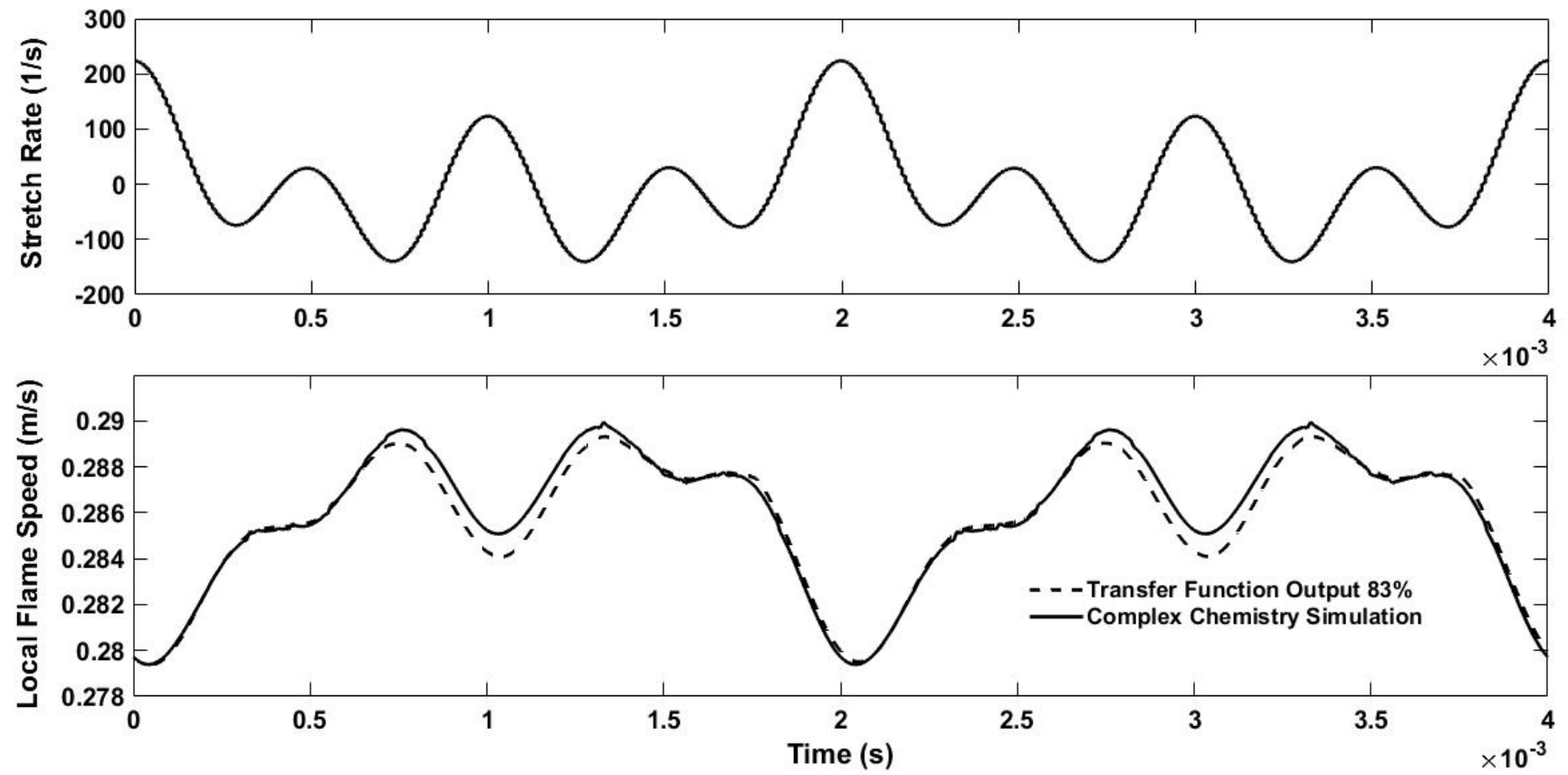

Fig. 9. Comparison of the transient response (complex chemistry) and the transfer function prediction of a laminar premixed flame to a random input in the high frequency range $(\varphi=0.8)$, Top figure: stretch rate perturbation as the input, Bottom figure: solid line - the transient response (complex chemistry) and dashed line - transfer function output (NARX-H) 

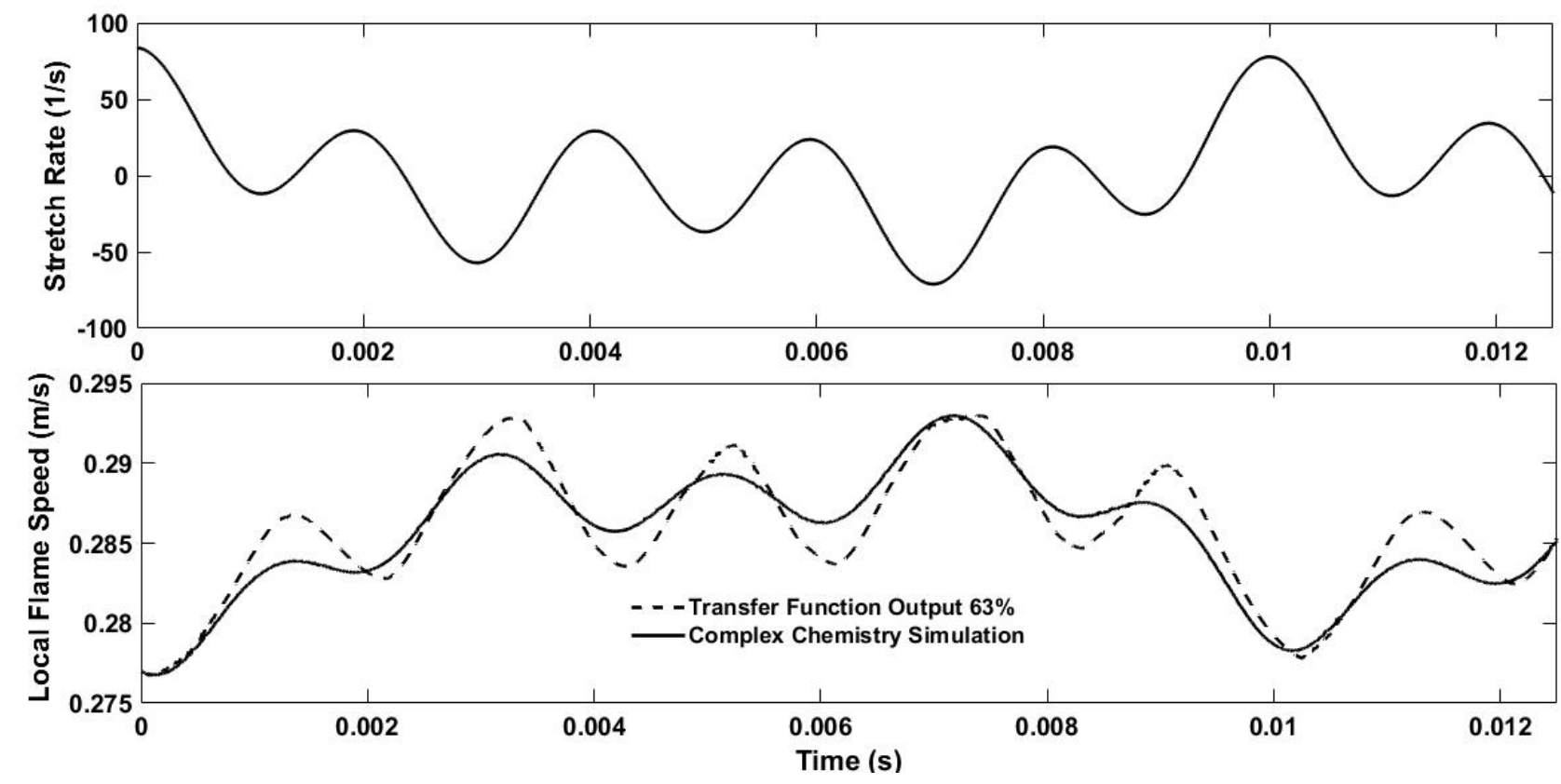

Fig. 10. Comparison of the transient response (complex chemistry) and the transfer function prediction of a laminar premixed flame to a random input in the combination of low and high frequency range $(\varphi=0.8)$, Top figure: stretch rate perturbation as the input, Bottom figure: solid line - the transient response (complex chemistry) and dashed line - transfer function output (NARX-A). 